\title{
PREVALENCE OF CD8/CD4 RATIO IN THE FETAL THYMIC PARENCHYME IN DOWN'S SYNDROME
}

\author{
Demetrio Tamiolakis ${ }^{1}$, Ioannis Venizelos ${ }^{2}$, Athanasia Kotini ${ }^{3}$, Sylva Nikolaidou ${ }^{1}$, \\ Nikolaos Papadopoulos ${ }^{4}$
}

General Hospital of Alexandroupolis: Department of Cytology ${ }^{1}$; Ippokration Hospital of Salonica: Department of Pathology²; Democritus University of Thrace: Department of Medical Physics ${ }^{3}$, Department of Histology - Embryology ${ }^{4}$

\begin{abstract}
Summary: Aim: The maturation of most T- lymphocyte precursors takes place within the meshwork of thymic epithelial cells. Different steps of this process can be defined by immunologic phenotyping. The prothymocytes are positive for the terminal deoxynucleotidyl transferase (TdT) and give rise to cortical thymocytes, which express CD1, CD2, CD3, CD5, and both CD4 and CD8. These CD4 and CD8 double-positive cortical thymocytes differentiate into two lineages: CD4+ or CD8+ lymphocytes of the thymic medulla, by the tenth week of gestation. Our study points towards the determination of the CD8 cytotoxic/suppressor capacity of the fetal thymus in Down's syndrome. Experimental design: A quantitative comparison of T-lymphocytes (CD3, CD4, and CD8) in the thymic parenchyme in embryos after voluntary abortion during $2^{\text {nd }}$ trimester of gestation and embryos with Down's syndrome, respectively, was performed. Results: Our results showed: 1) A statistically significant depletion in the total number of T-cells (CD3 positive) in the cases of embryos with Down's syndrome over those after voluntary abortion, during the second trimester of gestation $(p<0.0001, t$-test $) .2)$ A significant difference in the CD8/CD4 ratio in the cases of embryos with Down's syndrome, during the second trimester of gestation which was numerically stronger with the progress of fetal development $\left(20^{\text {th }}\right.$ week: $p<0.025 ; 24^{\text {th }}$ week: $p<0.01$, chi-square). Conclusions: The occurrence of increased CD8/CD4 ratio in the cases with Down's syndrome, in the second trimester of gestation, underlines the cytotoxic / suppressor property of the thymus in the affected fetuses.
\end{abstract}

Key words: Thymic T-cells; CD3; CD4; CD8; Down's syndrome; $2^{\text {nd }}$ trimester of gestation

\section{Introduction}

In the thymic cortex, massive numbers of densely packed small thymocytes (thymic lymphocytes) predominate, occupying the interstices of the epithelial reticulum, which in histological sections they largely obscure, and forming about $90 \%$ of the total weight of the thymus. A distinct subcapsular zone is present, housing the thymic stem cells, prothymocytes and lymphoblasts undergoing mitotic division. The first stem cells to enter the thymus in the embryo come from the yolk sac and liver during their haemopoietic phases, possibly, as in birds, being attracted by thymic chemotactic substances. During later periods it is probable that all thymic lymphocytes originate in the bone marrow, or at least have sojourned there, before passing in the bloodstream to the thymus. The cortex has two rather ill-defined zones: an outer cortex with a framework of Types 1-3 epitheliocytes and a deep cortex where Type 4 cells occur. Thymocytes undergo mitosis in all cortical zones as the clones of differentiating $\mathrm{T}$ cells mature, gradually moving deeper in the cortex. In rodents, cell cycling times of 8 hours have been recorded in the outer cortex, but no estimates exist for the human thymus. The appropriate conditions for the proliferation and differentiation of thymocytes appear to be produced by their close proximity to neighbouring epitheliocytes (9). Although the nature of these interactions is not clear, it may involve the release from the epitheliocytes of soluble mitogenic and differentiation factors as well as induction of changes through intercellular contact. During this process, thymocytes differentiate along the T-cell line, acquiring the CD3 + marker and T-cell receptors for antigen, and also switching into different $s u b$ population of $\mathrm{T}$ cells (3). The great range of different T-cell receptor types, running into many millions, is also established here by the expression of variable genes and related mechanisms (3).

As time passes, the differentiating thymocytes enter the medulla, and migrate through the walls of venules and lym- 
phatics to move into the circulation. Such cells (post-thymic thymocytes) are not immunocompetent within the cortex, and in general attain maturity only after entering the medulla or perhaps not until they reach their secondary lymphoid tissue destinations. However, the existence of antigen presenting cells and plasma cells in the medulla indicates that $\mathrm{T}$ lymphocytes can be activated within the thymus, if not in large numbers.

Four major lymphoid cell types are found in the thymic cortex, as determined by immunocytochemistry and flow cytometry; each of them has a different proportion of small, medium and large thymocytes. Firstly, there are cells which do not express the mature T-lymphocyte markers CD4 or CD8 (double negative cells) nor, initially, CD3. Most of these are large blast cells, which after undergoing mitosis become small, double negative thymocytes and begin to develop the T-cell receptor (TCR) complex and become CD3-positive. These blast cells are primarily located in the subcapsular cortex and around blood vessels, especially at the corticomedullary junction. During the development of the TCR the cells give a transient expression of g/d (gam$\mathrm{ma} /$ delta), followed by a/b (alfa/beta) and other TCR components (3), and simultaneously become double positive for CD4 and CD8, as well as the histochemical marker TdT (terminal deoxynucleotidyl transferase), characteristic of thymocytes thereafter (10). The majority of these doublepositive cells are small cortical thymocytes comprising $80-90 \%$ of the total thymocyte population. It is thought that the functional abilities of the T-cell repertoire is determined at this stage, with 'undesirable' lymphocytes dying in great numbers by apoptosis. The few that are rescued by the action of factors from the microenvironment (positive selection) become either CD4- or CD8positive (i.e. single rather than double positive); these cells are found in the medulla and are slightly larger than cortical thymocytes.

Medullary single-positive thymocytes may either be cells about to be exported to the periphery where they will become fully immunocompetent, or recirculating activated $\mathrm{T}$ cells which have entered the medulla secondarily (1). In addition, a few single positive cells represent early cortical cells that transiently express either CD4 or CD8 before becoming double-positive early thymocytes, as noted above.

In addition to these T-cell products, the thymus is also thought to be responsible for generating natural killer cell lineages.

In our settings we examined the differential expression of CD3, CD4, and CD8 in thymic medullary tissue obtained from fetuses with DS and controls after voluntary abortion, during the second trimester of development, in order to ascertain changes in the cell-mediated immunity of the organ in DS.

\section{Materials and methods}

Fetal thymus specimens were obtained from 20 fetuses; 10 cases after voluntary abortion and 10 cases with Down's syndrome at the second trimester of gestation (gestation week 20 and 24). Tissue samples were processed for paraffin section immunophenotyping and stained using the monoclonal antibodies CD3, CD4, and CD8 by DAKO.

Immunohistochemistry was performed using the «avidin - biotin - immunoperoxidase» technique. Sections were deparaffinized and the endogenous peroxidase activity was blocked by methanol with $0.3 \% \mathrm{H}_{2} \mathrm{O}_{2}$ (30 min, RT). Firststep $\mathrm{Ab}$ (antibody) (avidin-biotin) were diluted in TBS (Tris Buffer Saline, 0.05 M Tris in $0.15 \mathrm{M} \mathrm{NaCl}$ ) and $1 \%$ bovine serum albumin (BSA) and applied for $60 \mathrm{~min}$ (RT). After each step, the tissue was rinsed twice in TBS. For individual $\mathrm{Ab}$, the «antigenicity» was enhanced by antigen retrieval. Isotype-matched $\mathrm{Ab}$ were used as negative control. After incubation with first-step Ab, slides were washed in TBS and then incubated with appropriate second-step biotinylated $\mathrm{Ab}$ for $30 \mathrm{~min}$ (RT). Then, slides were washed and incubated with the avidin-biotin-complex for $30 \mathrm{~min}$ (RT) or with peroxidase-conjugated streptavidin. After washing, bound $\mathrm{Ab}$ were visualized by incubating in 3-amino-9-ethyl carbazole (AEC) for 5 to $10 \mathrm{~min}$. Sections were subsequently counterstained with hematoxyline and coverslipped.

The immunostained sections were examined with a X 40 objective and the distribution of CD3, CD4, and CD8 within the cell was recorded. Every stained cell was scored as positive regardless of staining intensity. To count the number of cells with CD3, CD4, and CD8 staining, a $10 \times 10$ square calibrated grid was inserted into the eyepiece of an Olympus BX40 binocular microscope.

Five-to-ten fields were examined for each section, and at least 1000 cells were scored, depending on cellularity.

\section{Results}

The sections were examined independently by two observers, and positive cellular staining for CD3, CD4, and CD8 antibodies was manifested as fine yellow cytoplasmic granularity and / or surface membrane expression.

\section{$2^{\text {nd }}$ Trimester of gestation:}

The thymic cortical and medullar substances of fetuses with Down's syndrome showed a depletion in the total number of T-cells as compared with controls after voluntary abortion, which was statistically significant $(\mathrm{p}<0.0001$, t-test). In the cases of Down's syndrome (Fig. 1), the average number was $108.6\left(43.4 \mathrm{CD} 3\right.$ positive cells $/ \mathrm{mm}^{2}$ (range 60 to 180 cells $/ \mathrm{mm}^{2}$ ). In the cases of voluntary abortions (Fig. 2), the average number was 235.3(73.7 CD3 positive cells $/ \mathrm{mm}^{2}$ (range 160 to 380 cells $/ \mathrm{mm}^{2}$ ).

\section{$20^{\text {th }}$ week of gestation:}

In the cases of Down's syndrome (Fig. 3), a significant increase in the CD8/CD4 ratio in comparison with the relevant ratio in the cases of voluntary abortions (Fig. 4), was shown (DS: CD8/CD4=10, Voluntary abortions: CD8/ $/ \mathrm{CD} 4=3, \mathrm{p}<0.025$, chi-square) 


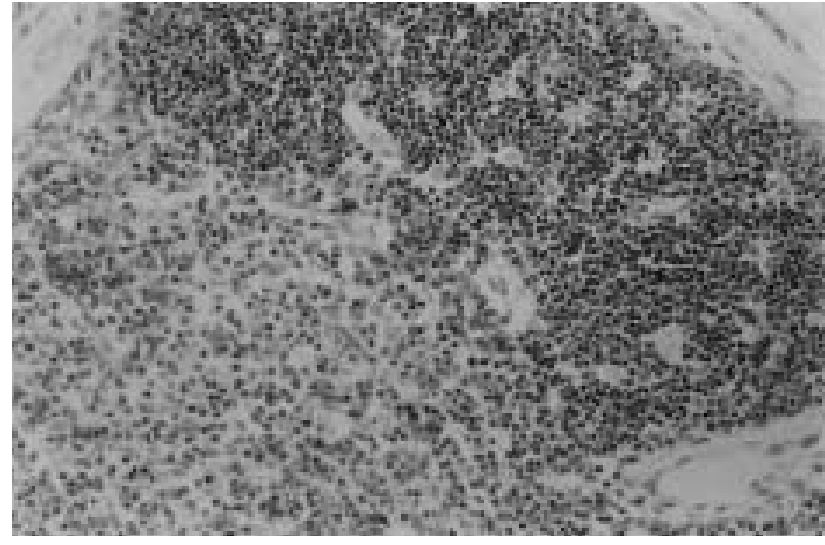

Fig. 1: Down's syndrome. T lymphocytic depletion in the thymic parenchyme during the second trimester of development. CD3 X100.

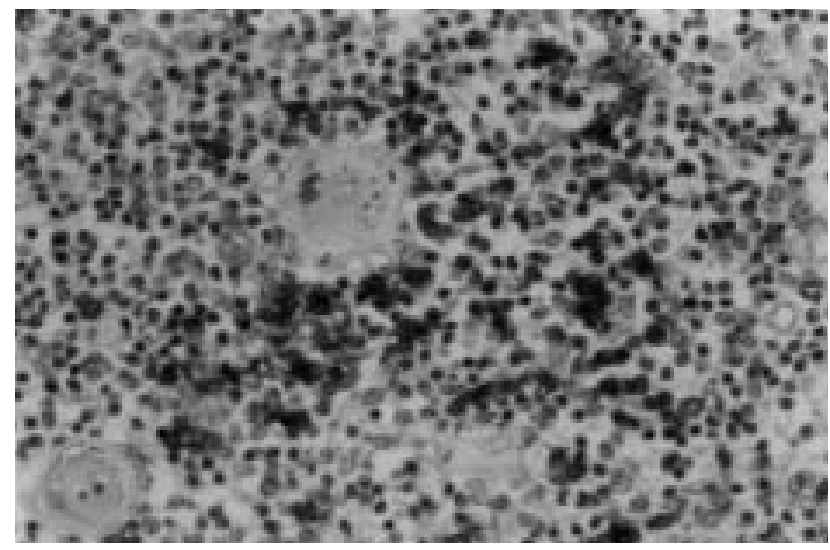

Fig. 3: Down's syndrome. Relative CD 8 lymphocytic predominance in the thymic parenchyme during the twentieth gestational week. CD8X200.

\section{$24^{\text {th }}$ week of gestation:}

In the cases of Down's syndrome, a stronger numerical CD8/CD4 ratio was observed in comparison with the $20^{\text {th }}$ week, while in the cases of voluntary abortions the relevant ratio remained constant (DS: $C D 8 / C D 4=15$, Voluntary abortions: $\mathrm{CD} 8 / \mathrm{CD} 4=3, \mathrm{p}<0.01$, chi-square).

The above CD3, CD4, and CD8 positive cells were demonstrated in perivascular sites of the cortex, the medulla, and the corticomedullary region, as well as around Hassal's corpuscles.

\section{Discussion}

Stem cells originate in the yolk sac, migrate through primitive lymphatics associated with blood islands, and populate the fetal liver, spleen, and bone marrow. These events occur during the second to third weeks of postconceptual life. Stem cells give rise to myeloid cells and lymphoid cells.

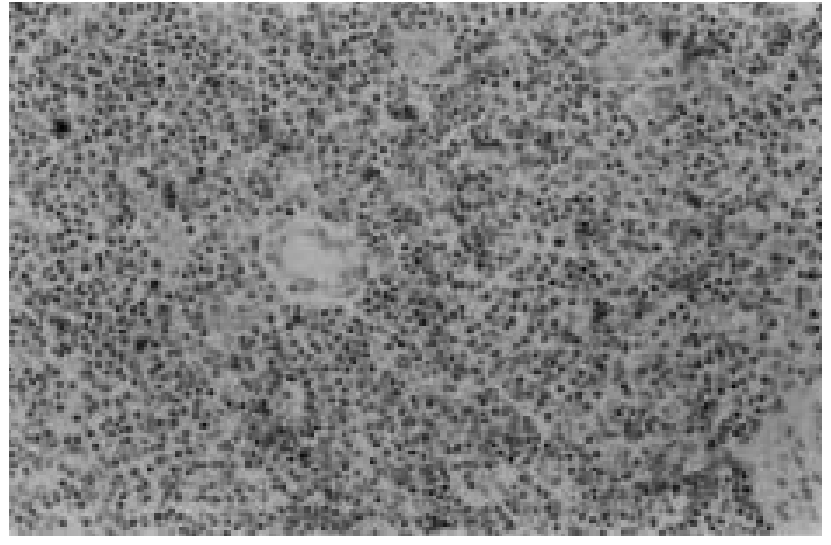

Fig. 2: Voluntary abortion. Distinct T lymphocytic distribution in the thymic cortical and medullar substrate during the twentieth gestational week. CD3X100.

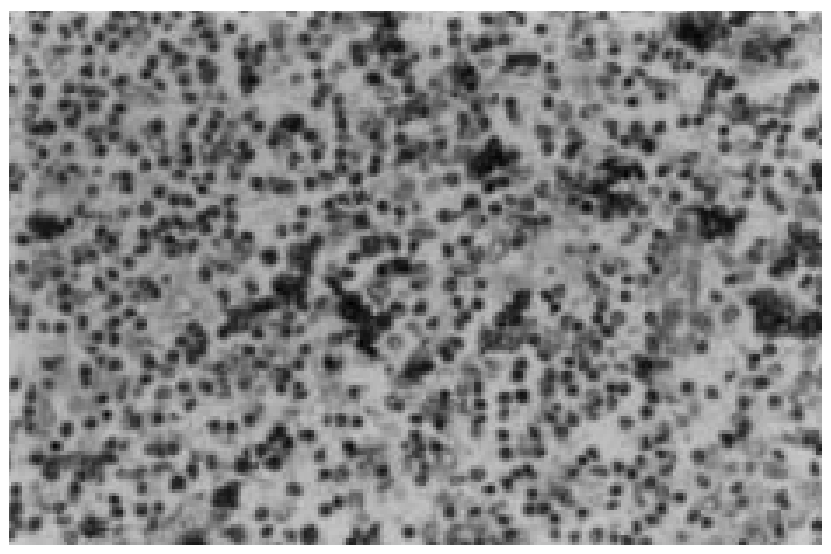

Fig. 4: Voluntary abortion. Distinct CD8 lymphocytic distribution in the thymic parenchyme during the twentieth gestational week. CD8X200.

Lymphoid cells consist of thymic dependent T cells, which are responsible for cell-mediated immunity, delayed hypersensitivity, and allograft rejection. B cells derived from the bursa equivalent, which probably is the fetal liver and bone marrow in man, differentiate into fully mature lymphocytes and plasma cells that synthesize and secrete antibodies. Cellular immunity ( $\mathrm{T}$ cells) and humoral immunity (B cells) are two complementary families of lymphoid cells. They coexist within organized lymphoid follicles that form the functional unit of the immune response.

Pre-T cells derived from the fetal liver and bone marrow home to the subcapsular region of the thymic cortex $(5,8)$. Pre-T cells from the bone marrow are transformed within the epithelial thymic microenvironment, where they are educated to distinguish self from nonself. This developmental event involves both negative and positive selection. $\mathrm{T}$ cells that react in a destructive fashion with host antigens are eliminated. Selection favors $\mathrm{T}$ cells that cooperate with 
B cells and macrophages to achieve complementary T-cell and B-cell immunologic functions. T-cell subsets are identified by the use of monoclonal antibodies. A large type I blast cell in the subcapsular region strongly expresses terminal deoxynucleotidyl transferase (TdT). A smaller type II cell accounts for $60 \%$ to $70 \%$ of the cortical thymocytes. These cells express CD4 and CD8 receptors in addition to TdT and CD38. A third type of thymocytes expresses the CD3 complex in the medulla. It is strongly positive for HLA-A, $-\mathrm{B}$, and $-\mathrm{C}$ markers. The ratio of helper and suppressor cells in tissue is similar to that in peripheral blood. Thymic epithelial cells also secrete hormones such as thymulin and thymopoietin (6).

Apoptosis or programmable cell death is the fate of more than $90 \%$ of thymocytes, and most die in situ. The remaining $10 \%$ of thymocytes circulate to the T cell-dependent regions of the lymph nodes, spleen and MALT. Maturation takes place in the thymic medulla, where cells are marked as T4 helper or T8 suppressor cells. Strong T-cell suppressor activity predominates in the first year of life $(7,13)$ and suppresses fetal immunoglobulins.

Down's syndrome (DS) is associated with cellular and humoral immunological abnormalities and modified histology of the lymphoid and epithelial compartments of the thymus has been reported $(2,4,11,12)$. Fonseca et al (4) investigated the extracellular matrix (ECM) of DS thymuses and they suggested that severe intrathymic ECM structures changes occurring in DS may be linked to abnormal cortical thymocyte depletion.

In our study we confirmed previous reports of thymocytes depletion in Down's syndrome. Moreover, the prevalence of CD8/CD4 ratio in fetuses with Down syndrome over controls after voluntary abortion in the second trimester of gestation directs towards a cytotoxic / suppressor capacity of the thymic parenchyme in the affected fetuses.

\section{References}

1. Agus D B, Surh C D, Sprent J. Reentry of T cells to the adult thymus is restricted to activated T cells. J Exp Med 1991;173:1039-46.

2. Aita M, Franze A, Gabrielli F. S-100 immunoreactive interdigitating cells in normal and in Down's syndrome human thymuses. Cell Biol Int Rep 1991;15(8): 645-59.

3. Bannister L. Haemolymphoid System In: Gray's Anatomy $38^{\text {th }}$ Edition, Churchill Livingstone 1995, chapter 9:1420-1.

4. Fonseca EC, Lannes-Vieira J, Villa-Verde DM, Savino W. Thymic extracellular matrix in Down's syndrome. Braz J Med Biol Res 1989;22(8):971-4.

5. Ford CE. Traffic of lymphoid cells in the body. In Wolstenholme G, Porter R, editors: The thymus: experimental and clinical studies, Boston: Little, Brown, 1966:131.

6. Goldstein G. Thymus factors in immunity. Ann N Y Acad Sci 1975;249:177.

7. Haynes BF, Denning SM, Singer KH, Kurtzberg J. Ontogeny of T-cell precursors. Immunol Today 1989;10:87.

8. Janossy G, Bofill M, Trejdosiewicz LK et al. Cellular differentiation of lymphoid subpopulations and their microenvironments in the human thymus. In MullerHermelink HK, editor: The human thymus: histophysiology and pathology, Berlin: Springer Verlag 1986:89.

9. Janossy G, Prentice HG, Grob JP et al. T lymphocyte regeneration after transplantation of $\mathrm{R}$ cell depleted allogeneic bone marrow. Clin Exp Immunol 1986;63: $577-86$

10. Janossy G, Campana D. Ontogeny of the human T cell receptors-single cell studies. Thymus Update 1989;2:39-58.

11. Larocca LM, Lauriola L, Ranelletti FO et al. Morphological and immunohistochemical study of Down syndrome thymus. Am J Med Genet Suppl 1990; 7:225-30.

12. Medvedev Niu, Popova ED. Thymus morphology in Down's syndrome. Arkh Patol 1982;44(6):27-30

13. Papadogiannakis N, Svend-Aage J, Olding LB. Suppressor cell activity in human cord blood. In: Chaouat G, editor: The immunology of the fetus, Boca Raton, FL, CRC Press, 1990:215.

Submitted March 2003.

Accepted June 2003.

Nikolaos Papadopoulos, Assoc. Professor in Histology-Embryology, Democritus University of Thrace. Dragana, 68100 Alexandroupolis, Greece. e-mail: npapad@med.duth.gr 